\title{
Evaluación financiera del Banco del Austro y análisis del impacto de las regulaciones bancarias decretadas por el Gobierno Ecuatoriano en el periodo 2010-2014
}

DOI: https://doi.org/10.33262/ap.v3i3.1.84

\begin{abstract}
(c) (1) (2)
Financial evaluation of Banco del Austro and analysis of the impact of the banking regulations decreed by the Ecuadorian Government in the period 2010-2014
\end{abstract}

Edisson Israel Guerrero Freire. ${ }^{1}$, Andrea Alejandra Santana Torres. ${ }^{2}$, Sixto Santiago Ibáñez Jacome. ${ }^{3}$ \& Luz Irene Flores Abarca. ${ }^{4}$

\begin{abstract}
.
Introduction. Government policies remain present in each of the spheres of a territory. They have the objective of providing practical solutions to the problems of the Society. However, the political ideology of the leaders can lead to a notorious change, making the policies enacted can benefit a social group and affect others. These policies are classified as regulatory, distributive, redistributive, and constitutive or institutional. Objective. Carry out an analysis of the economic policies established by the government and their impact on the financial statements of Banco del Austro in the period 2010-2014. Methodology. The research has a mixed approach, an exploratory, descriptive and correlational research is carried out. The population was made up of the directors of the

\footnotetext{
${ }^{1}$ Universidad Técnica Luis Vargas Torres, Facultad de Ciencias Administrativas y Económicas, Sede Santo Domingo, Ecuador, edisson.guerrero@utelvt.edu.ec, ORCID 0000-0003-3101-4029

${ }^{2}$ Universidad Técnica Luis Vargas Torres, Facultad de Ciencias Administrativas y Económicas, Sede Santo Domingo, Ecuador, andrea.santana@utelvt.edu.ec, ORCID 0000-0002-6045-6216

${ }^{3}$ Universidad Técnica Luis Vargas Torres, Facultad de Ciencias Administrativas y Económicas, Sede Santo Domingo, Ecuador, sixto.ibañez@utelvt.edu.ec, ORCID 0000-0002-3953-5980

${ }^{4}$ Universidad Técnica Luis Vargas Torres, Facultad de Ciencias Administrativas y Económicas, Sede Santo Domingo, Ecuador, luz.flores@utelvt.edu.ec, ORCID 0000-0002-6919-4363
} 
Banco del Austro, and a sample of 28 people was used to apply the interview. Results. It was determined that government policies issued in the 2010-2014 period affect the financial statements, and had a negative impact, since they reduced their liquidity, going from a liquidity of 0.31 to 2014 to a liquidity of 0,28 since 2010 . It can be seen that five resolutions issued by the government are classified as regulatory, two as redistributive and one is classified as distributive. Conclusions. The economic policies adopted by the government in the 2010-2014 period negatively affected the financial statements of Banco del Austro.

Keywords: Government Policies, Economic Profitability, Financial Profitability, Financial Indicators, Finance.

\section{Resumen.}

Introducción. Las políticas gubernamentales permanecen presentes en cada una de las esferas de un territorio. Tienen el objetivo de dar soluciones prácticas a las problemáticas de la Sociedad. Sin embargo, la ideología política de los mandatarios puede conllevar un cambio notorio, haciendo que las políticas decretadas puedan benefician a un grupo social y afectar a otros. Estas políticas se catalogan en regulatorias, distributivas, redistributivas y constitutivas o institucionales. Objetivo. Realizar un análisis de las políticas económicas instaurada por el gobierno y de su incidencia en los estados financieros del Banco del Austro en el periodo 2010-2014. Metodología. La investigación tiene un enfoque mixto, se lleva una investigación exploratoria, descriptiva y correlacional. La población fue conformada por los directivos del Banco del Austro, y se utilizó una muestra de 28 personas para aplicar la entrevista. Resultados. Se determino que las políticas gubernamentales emitidas en el periodo 2010-2014 inciden sobre los estados financieros, y tuvieron un impacto negativo, puesto que redujeron su liquidez pasando desde el año 2010 de una liquidez de 0,31 al año 2014 a una liquidez de 0,28. Se aprecia que cinco resoluciones emitidas por el gobierno se clasifican como regulatorias, dos como redistributiva y una se clasifica como distributiva. Conclusiones. Las políticas económicas adoptadas por el gobierno en el periodo 2010-2014 afectaron negativamente en los estados financieros del Banco del Austro.

Palabras claves: Políticas Gubernamentales, Rentabilidad Económica, Rentabilidad Financiera, Indicadores Financieros, Finanzas.

\section{Introducción.}

Las políticas gubernamentales son generadas con la iniciativa de la atención positiva de los inconvenientes de la sociedad, sin embargo, paralelamente estas benefician a unos y afectan a otros. En esta situación especial el perjudicado es el Banco del Austro puesto que en el lapso de estudio han surgido políticas públicas que han perjudicado los valores de productividad del Banco. Al reducir la productividad de la organización anteriormente mencionada, en la misma hay problemas para consumar las metas a corto y largo plazo. 
Hallar estudios previos a este, donde se trate el asunto de las políticas gubernamentales en el área bancario es poco recurrente. Por tal motivo se hace referencia a trabajos investigativos que traten el asunto de las políticas gubernamentales y su incidencia en otros sectores.

En el trabajo llevado a cabo por Rodríguez y Yépez (2014) titulado "Análisis de las políticas públicas dirigidas a la inserción socio-laboral de individuos con discapacidad" de la Universidad Central del Ecuador que tiene como fin decidir los componentes que inciden en el cumplimiento o incumplimiento de las Políticas Públicas en relación a la Inserción Socio-Laboral de los individuos con discapacidad en Ecuador se concluye que:

La creación y ejecución de Políticas Públicas, para la prevención, atención $e$ inclusión de las personas con discapacidad en el país, con la correspondiente asignación de recursos económicos a través de planes, programas, campañas y proyectos en beneficio de estas personas, se orienta al mejoramiento y cohesión social de este grupo... (Rodriguez Montenegro \& Yépez Andino, 2014)

Otro de los trabajos revisados es el de la creadora Quishpe (2014) que lleva por título "La política pública de ayuda social por medio del crédito de desarrollo humano y su incidencia en las condiciones de vida de los beneficiarios" de la Universidad Técnica de Ambato el cual tiene como fin primordial decidir la interacción de la Política Pública de ayuda social por medio del Crédito de Desarrollo Humano, en las condiciones de vida de los beneficiarios, para el mejoramiento de la calidad de vida. En este trabajo la creadora llega a la misma conclusión que las autoras del trabajo anterior. Esto se prueba ya que la misma dice que: La Política Pública en términos de subsidios ha mejorado las condiciones de vida de los beneficiarios... (Quishpe Quispe , 2014).

Además, se verificó el trabajo "Análisis y evaluación del efecto de la resolución \# 116 del comité de negocio exterior (Comex) aplicadas a las importaciones en el Ecuador” de la ingeniera Jaramillo (2015) eligiendo el título de magister en gestión de organizaciones de la Universidad Católica y su objetivo es: Examinar y evaluar el efecto de la resolución \#116 en las importaciones de alimentos procesados en el territorio, la creadora concluye que:

La resolución 116 entra en vigencia en diciembre del 2013, y establece el control previo de las importaciones para 293 productos. El objetivo de la resolución es, en primera instancia, dar cumplimiento al mandato constitucional de protección al consumidor por parte del Estado (en términos de calidad), con objetivos secundarios relacionados a la política pública de cambio de matriz productiva, especialmente en su estrategia de sustitución de importaciones; así como el efecto resultante de ayudar en el equilibrio de la balanza comercial. (Jaramillo Bernita, 2015).

Al final, se comprobó el trabajo de los autores Jami y Karolys (2012) llevado a cabo en la Universidad Técnica de Cotopaxi con el título "La falta de aplicación de políticas de 
estado en el interior de rehabilitación social de Latacunga" empero con la particularidad de que el asunto del mismo es la carencia de políticas públicas en el interior de rehabilitación social de Latacunga. En este trabajo los autores concluyen que: Las Políticas de Estado referente a esta acción son ineficientes lo cual afecta de manera directa en el proceso de Reinserción Social y por ende en la Reincidencia de Delitos. (Jami Negrete Héctor Adeodato \& Karolys Tovar , 2012).

Debido a esta conclusión los autores de dicha investigación recomiendan que "es necesario impulsar las Políticas de Estado en cuanto a la Rehabilitación Social en los diferentes instrumentos legales existentes a fin de garantizar el verdadero objeto de la Rehabilitación Social y su función de Reinserción" (Jami Negrete Héctor Adeodato \& Karolys Tovar, 2012).

Se concluye tras revisar las investigaciones antes mencionadas que: Las políticas públicas tienen el objetivo de mejorar la situación existente en una determinada entidad o sector. Esta conclusión es basándose en el punto de vista del gobierno. Pero desde el punto de vista de la otra parte (Banco del Austro) las políticas gubernamentales económicas, provocan un decremento de la rentabilidad en el Banco, así como de otros indicadores financieros (estabilidad y eficiencia).

Con el objetivo de elevar la calidad del análisis de casos y enriquecer los conocimientos de algunos aspectos se consultaron algunas bibliografías. De estas se extrajeron algunos conceptos que se mostraran a continuación:

\section{Bienestar social}

El punto de vista de Allardt encierra aspectos fundamentales de la sociedad. Este autor define el bienestar social como "una consideración más completa de las condiciones necesarias para el desarrollo humano. Un enfoque sobre las necesidades básicas se concentra en las condiciones sin las cuales los seres humanos no pueden sobrevivir, evitar la miseria, relacionarse con otras personas y evitar el aislamiento" (pág. 36).

El autor del presente estudio de casos define la paz social como el cubrimiento de las necesidades simples y la paz ciudadana sin estar alejado de la sociedad.

\section{Calidad de vida:}

El autor (Cabedo, 2003) plantea acerca de la calidad de vida que "Es la satisfacción de las necesidades de la sociedad, basado en una sociedad de democracia, igualdad, leyes y justicia siendo la base del desarrollo social y equitativo" (pág. 56).

\section{Desarrollo}

Existen diversos aspectos acerca del desarrollo, la ONU plantea un criterio bastante destruido sobre que es desarrollo basándose prácticamente en el punto de vista de las personas. 
El desarrollo en general es básicamente un proceso de vida que permite contar con alternativas u opciones de selección para las personas. Las aspiraciones de las personas pueden ser muchas, pero fundamentalmente se refieren a tres: (a) la búsqueda de conocimientos; (b) la posibilidad de tener una vida prolongada y saludable; y (c) tener acceso a los recursos que permitan un aceptable nivel de vida. Es a partir de estas tres finalidades que se derivan muchas otras. (ONU, 1990).

Por lo cual se puede concluir que desarrollo no es más que tener la probabilidad de brindar a la sociedad cualquier servicio o producto con óptima calidad y además tener bajos índices de indicadores sociales (pobreza, analfabetismo...).

\section{Desarrollo empresarial:}

Para los autores (Arencibia \& Cardero, 2014) el desarrollo empresarial no es más que "el desarrollo empresarial es un proceso que relaciona la realidad organizativa con su entorno y esta a su vez involucra todos los recursos ya sean humanos, técnicos y financieros con los cuales cuenta la organización" (pág. 45).

\section{Desarrollo socioeconómico}

El desarrollo socioeconómico es una de las vertientes del desarrollo de forma general, y a partir de la perspectiva del creador del presente estudio de casos una de las más relevantes. Paralelamente tiene la particularidad que se puede examinar de numerosas perspectivas. Por lo cual el creador (Núñez, 2004) da una definición de desarrollo socioeconómico con base en la calidad de vida "a través de la correcta explotación de los recursos existentes en el País da apertura a obtener una mejor calidad de vida de las personas que habitan en un mismo territorio" (pág. 56).

Otro autor (Sánchez Pérez, 2004) en el mismo año, define el desarrollo socioeconómico desde la vertiente de los bienes sociales y enfocados al pueblo planteando que "el desarrollo socioeconómico es la búsqueda de un bien social y económico de un país hacia sus habitantes, A través de la explotación total y adecuado de los ingresos” (pág. 46).

También se puede definir el desarrollo socioeconómico desde el punto de vista Gubernamental y así lo define (Schaffer R. , 2000). Planteando que desarrollo socioeconómico es "un objetivo del Gobierno puesto que implica alcanzar el bienestar social que se encuentra relacionado con el desarrollo económico ya que para satisfacer las necesidades de la sociedad implica comprar bienes y Servicios" (pág. 67). Resumiendo, se puede asegurar que desarrollo socioeconómico es conseguir la igualdad económica y social de un territorio, administrando correctamente las ganancias permanentes y aprovechando eficientemente los no permanentes.

\section{Desarrollo económico:}

Para el autor (Boisier, 1999), el desarrollo económico es "el desarrollo económico es un proceso continuado cuyo mecanismo esencial consiste en la 
aplicación reiterada del excedente en nuevas inversiones, y que tiene como resultado la expansión incesante de la unidad productiva de que se trate. Esta unidad puede ser desde luego una sociedad entera” (pág. 28).

\section{Políticas Gubernamentales (Publicas):}

Hay diversos autores que han dado conceptos sobre que son políticas gubernamentales o públicas. Los cuales poseen diversos puntos de vista en común empero difieren en ciertos puntos que se especificaran en el resumen. El primer caso es el del autor (Knoepfel, 2007). el cual indica que:

Una política pública se define como "una concatenación de decisiones o de acciones, intencionalmente coherentes, tomadas por diferentes actores, públicos y ocasionalmente privados cuyos recursos, nexos institucionales $e$ intereses varían a fin de resolver de manera puntual un problema políticamente definido como colectivo. Este conjunto de decisiones y acciones da lugar a actos formales, con un grado de obligatoriedad variable, tendientes a modificar el comportamiento de grupos sociales que, se supone, originan el problema colectivo a resolver (grupos objetivo), en el interés de grupos sociales que padecen los efectos negativos del problema en cuestión (beneficiarios finales). (pág. 20)

En conclusión, se puede evidenciar que las políticas públicas son acciones, decisiones o respuestas determinadas por el estado para solucionar un problema existente o resolver situaciones puntuales.

\section{Ciclo de las políticas públicas:}

Para la autora (Delgado Godoy ,2009) el ciclo de las políticas se divide en 5 momentos los cuales se describirán a continuación:

\section{La identificación de problemas.}

El gobierno advierte la existencia de problemas u oportunidades en la sociedad y se plantea si debe actuar o no. Esta fase incluye en primer lugar, actividades de detección y selección de cuestiones, y, en segundo lugar, actividades de definición de problemas. (pág. 61)

\section{La formulación de las políticas.}

Una vez que el gobierno conoce la existencia de un problema, lo define y rechaza la opción de no actuar sobre él, comienza la fase de formulación de políticas, que tiene que ver con el desarrollo de cursos de acción (alternativas, propuestas, opciones) aceptables y pertinentes para enfrentarse a los problemas públicos. Las políticas son concebidas, por lo tanto, como soluciones a los problemas públicos. Esta fase incluye las siguientes actividades: (Delgado Godoy , 2009) 
1) El establecimiento de metas y objetivos a alcanzar.

2) La detección y generación de alternativas que permitan alcanzar los objetivos.

3) La valoración y comparación de las alternativas.

4) La selección de una opción o combinación de ellas.

\section{La adopción de la decisión.}

Esta fase está exclusivamente en manos de uno o varios decisores públicos: para que una política sea considerada pública ésta debe haber sido generada por medios gubernamentales, debe emanar de una autoridad pública. Otras fases del ciclo de las políticas públicas (la implantación, por ejemplo) pueden quedar parcialmente en manos de actores, individuos u organizaciones que no están investidos de autoridad pública: no es el caso de la fase de adopción de la decisión. (Delgado Godoy, 2009)

\section{La implantación de las políticas públicas.}

Esta fase comienza una vez adoptada la decisión, y comprende todas las actividades y procesos desarrollados hasta que aparecen los efectos asociados con la intervención pública en cuestión. En ella las unidades administrativas correspondientes movilizan recursos económicos y humanos, sobre todo, para poner en práctica la política adoptada. Se trata de la puesta en marcha o ejecución de las políticas. (Delgado Godoy , 2009)

\section{La evaluación de las políticas.}

"Las unidades de evaluación de la administración pública determinan en qué medida se han logrado los objetivos de la política pública en cuestión. La evaluación cierra el ciclo de las políticas, y puede retroalimentar el proceso en cualquiera de sus fases. En la práctica, la evaluación no está tan extendida como sería deseable" (Delgado Godoy , 2009).

\section{Ventajas de las políticas públicas:}

Las Políticas Públicas tienen la potencialidad de resolver problemas concretos, de promover integración social: es decir, permitir que la gente viaje en el "mismo avión." Este último término hace referencia también a la búsqueda de la equidad, ya que, si bien es cierto que una propuesta de política puede beneficiar a unos y perjudicar a otros, se debe tener en mente a la mayoría que es quien decide en una democracia, sin menospreciar claro está, a las minorías. (Ruiz Lopez \& Cadenas Ayala, 2013).

\section{Desventajas de las políticas públicas:}

Las Políticas Públicas que son producto de las decisiones de los gobiernos tienden a no representar a los intereses y puntos de vista de los distintos 
sectores de la sociedad. Reafirmando el predominio de uno de los actores sobre otro, protegiendo siempre sus intereses, lo que lleva solamente a una alta probabilidad de fracaso en la implementación de las mismas. (Ruiz Lopez \& Cadenas Ayala, 2013).

\section{Distributivas:}

Para (Delgado Godoy , 2009) las políticas distributivas son "las políticas distributivas consisten en proporcionar bienes o servicios a determinado segmento de la población (individuos, grupos, empresas, etc.): las actuaciones públicas consistentes en dar asesoramiento jurídico a las pequeñas y medianas empresas constituyen una política distributiva" (pág. 63).

\section{Regulatorias:}

(Delgado Godoy , 2009) expone que "las políticas regulatorias imponen restricciones o limitaciones al comportamiento de individuos y grupos, como el código de circulación" (pág.64).

\section{Redistributivas:}

(Delgado Godoy , 2009). Las define como:

Son un esfuerzo deliberado del gobierno por cambiar la asignación de riqueza, ingresos, propiedades o derechos entre amplios grupos o clases sociales. Son las políticas más características del Estado del bienestar; en este sentido, uno de los ejemplos más conocido viene dado por los sistemas de seguridad social. (pág.65)

\section{Constitutivas o institucionales:}

(Olavarría Gambi, 2007). Las define como las políticas institucionales tienen que ver con las leyes, reglamentos y procedimientos involucrados para tomar la decisión sobre la propuesta e implementarla. Estas políticas pueden inhibir el surgimiento o puesta en práctica de ciertas iniciativas o propuestas, simplemente porque son contrarias al diseño institucional. (pág.46)

\section{Finanzas}

El término finanzas es un término muy debatido por diferentes autores, por lo que en el presente análisis de casos se hará énfasis en dicho concepto.

Para (Andrade, 2005) las finanzas son:

Área de actividad económica en la cual el dinero es la base de las diversas realizaciones, sean estas inversiones en bolsa, en inmuebles, empresas industriales, en construcción, desarrollo agrario. Es el área de la economía 
en la que se estudia el funcionamiento de los mercados de capitales y la oferta y precio de los activos financieros. (pág. 293)

Otro autor (Bodie \& Merton , 2003) la conceptualizan de manera más resumida planteando que "Las finanzas estudian la manera en que los recursos escasos se asignan a través del tiempo" (pág. 2). Después de plantear el concepto dado por varios autores el autor del presente análisis de casos plantea que la finanza no es más que todas las actividades que lleva una empresa referente al dinero tanto para obtenerlo como para pagarlo.

\section{Finanzas corporativas:}

Para definir esta rama de las finanzas se vio el autor (Azofra, 2013) el cual plantea que:

La rama de las finanzas que analiza las decisiones financieras tomadas dentro de las empresas y los criterios e instrumentos utilizados para tomar estas decisiones. Es también común asociar el término de finanzas corporativas a las actividades de la banca de inversión relacionadas con los procesos de concentración empresarial, fusiones absorciones $y$ adquisiciones. (Pág.135-166).

\section{Finanzas públicas:}

El primer autor al que se hace referencia es (Conchas, 2001), este plantea acerca de las finanzas públicas que son "las finanzas públicas son el estudio de los efectos de los presupuestos sobre la economía y, en particular, de su influencia en la consecución del mayor de los objetivos económicos, a saber, el desarrollo, la estabilidad, la equidad y la eficiencia de la economía" (pág. 16).

\section{Análisis económico financiero}

Para tener una mejor idea de que es análisis económico financiero se dividirá el concepto en dos términos: Análisis económico y Análisis financiero.

\section{Análisis económico:}

Para el autor (Derkach , 1986).el análisis económico lo define como:

El Análisis Económico constituye un medio imprescindible para el control rutinario de la marcha del cumplimiento del plan económico y el estudio de los resultados de la actividad económica - productiva de las empresas y sus dependencias estructurales para caracterizar la marcha del cumplimiento del plan, se estudian sus indicadores, los datos de la contabilización y los balances, así como otros datos de consulta. (pág. 50).

\section{Análisis financiero}

(Baena Toro, 2010) Plantea que el análisis financiero es: 
Un proceso de recopilación, interpretación y comparación de datos cualitativos y cuantitativos, y de hechos históricos y actuales de una empresa. Su propósito es el de obtener un diagnóstico sobre el estado real de la compañía, permitiéndole con ello una adecuada toma de decisión. (pág.77)

También existe otro concepto del mismo tema planteado por otro autor Horngren (1996). Este autor basa su concepto en el análisis personal, mientras que Baena (2010) se enfoca más en el proceso en sí. (Horngren, Contabilidad de costos : un enfoque gerencial, 1996) Plantea que "el Análisis Financiero, es la emisión de un juicio criterio u opinión de la información contable de una empresa, por medio de técnicas o métodos de análisis que hacen más fácil su comprensión y presentación" (pág. 154) Por lo que para el autor del presente análisis de casos a modo de resumen plantea que el análisis económico financiero no es más que la forma fundamental de control y además de emitir un juicio acerca de la información contable.

\section{Indicadores financieros:}

Para (Foulke, 1973) los indicadores financieros "Son aquellos necesarios para presentar una información completa de los eventos económicos de una entidad durante un período determinado y a una fecha dada" (pág. 94)

(Vásquez, Guerra , \& Ahmed., 2008).) Tienen otro concepto más extenso de indicadores financieros en el cual ellos plantean que:

Son el producto de establecer resultados numéricos basados en relacionar dos cifras o cuentas bien sea del Balance General y/o del Estado de Pérdidas y Ganancias. Los resultados así obtenidos por sí solos no tienen mayor significado; sólo cuando son relacionados unos con otros y son comparados con los de años anteriores o con los de empresas del mismo sector y a su vez el analista se preocupa por conocer a fondo la operación de la compañía, se pueden obtener resultados significativos y sacar conclusiones sobre la situación financiera real de la empresa” (pág. 44).

Por lo que a modo de conclusión los indicadores financieros son una relación entre datos extraídos de los estados financieros con el objetivo de conocer la situación financiera de la empresa del pasado y del presente para ayudar a la toma de decisiones futuras.

\section{La rentabilidad económica:}

Para los autores (Braley \& Myers, 2002) la rentabilidad económica es "el motor del negocio y corresponde al rendimiento operativo de la empresa. Se mide por la relación entre la utilidad operativa, antes de intereses e impuestos, y el activo o la inversión operativa" (pág.87).

Por lo que se puede concluir que la rentabilidad económica es el beneficio que han generado los activos de una empresa por cada unidad de dinero invertida. 


\section{Rentabilidad sobre los activos (ROA)}

Es importante ver el concepto planteado por los autores (Cortés Romero, Rayo Cantón, \& Lara Rubio, 2011) que dice que "la rentabilidad financiera es una medida de rentabilidad empresarial que refleja el rendimiento obtenido por la empresa con el dinero invertido por los accionistas" (pág. 6).

En resumen, la rentabilidad financiera no es más que un sinónimo de lo que es rentable o lo que es lo mismo generar provecho, utilidad, ganancia o beneficio.

\section{Rentabilidad sobre el Patrimonio o capital invertido (ROE):}

Los autores (Ruiz Porras \& Steinwascher, 2007) definen este indicador como:

La razón de ingreso a neto al capital contable; esto es, un indicador del rendimiento sobre la inversión de los accionistas. Finalmente, el margen neto es la razón del ingreso neto a las ventas netas, esto es, un indicador de la facilidad de convertir ventas en utilidad. (pág. 5)

La relación que existe entre el ROE y la ROA determina el efecto de apalancamiento:

Esto puede ser de 3 formas diferentes:

- Positivo, que se produce cuando el ROE es superior al ROA.

- Nulo (o cero), cuando ambas ratios coinciden.

- Negativo, cuando el ROE es inferior al ROA.

Es importante también analizar dentro de la rentabilidad dos factores fundamentales que son:

\section{Utilidades netas:}

(Aguilera, 2000) La define como la mejor expresión de resultado de un sistema empresarial. Ellas se constituyen en la máxima garantía que permite remunerar y continuar remunerando los públicos internos y externos de la organización. (pág. 58)

Desde un punto de vista cualitativo el autor Aguilera (2000) define las utilidades netas con un concepto bastante completo, pero no hace referencia a nada desde el punto de vista cualitativo por lo que es necesario revisar lo que plantean los autores (Fontalvo Herrera, Mendoza Mendoza , \& Morelos Góme, 2011)

La utilidad neta es igual a las ventas netas menos el costo de ventas, menos los gastos operacionales, menos la provisión para impuesto de renta, más otros ingresos menos otros gastos. Esta razón por sí sola no refleja la rentabilidad del negocio. Determina el porcentaje que queda en cada venta después de deducir todos los gastos incluyendo los impuestos. (pág. 320) 


\section{Utilidades brutas:}

(Fontalvo Herrera T. J., 2014) La define como "la utilidad propia de los servicios que se prestan o los productos que se elaboran” (pág. 61).

Desde el punto de vista cuantitativo los autores (Horngren, Sundem, \& Stratton, Contabilidad admnistrativa. 13ra edicion, 2006) la definen como el exceso de las ventas sobre el costo de los artículos vendidos; es decir, el costo de la mercancía que se adquiere o fabrica y luego se vende" (pág. 64)

Utilidad Bruta $=$ Ventas netas de la empresa - Costo de ventas durante el ejercicio contable.

\section{Enfoque:}

En el presente análisis de casos se utiliza el enfoque cuantitativo pues los datos que se utilizaran una parte de ellos son numéricos pues son los datos de los estados financieros del Banco del Austro los cuales se utilizarán para el análisis económico financiero.

Pero también existe otro enfoque en el presente análisis de casos que es el cualitativo que el mismo autor lo define como: "El enfoque cualitativo utiliza recolección de datos sin medición numérica para descubrir o afinar preguntas de investigación y puede o no probar hipótesis en su proceso de interpretación” (Hernández, Fernández , \& Baptista, 2003, pág. 61).

Este enfoque se utilizará en la descripción de las políticas gubernamentales que afectan los indicadores financieros del Banco del Austro.

\section{Investigación bibliográfica:}

El autor Tamayo (2004,) describe la investigación bibliográfica como "la investigación bibliográfica es la que se realiza con base en la revisión de documentos, manuales, revistas, periódicos, actas científicas, conclusiones de simposios y seminarios o cualquier tipo de publicación considerado como fuente de información”. (pág. 84).

En este análisis de caso se utilizó la investigación bibliográfica para determinar los conceptos relacionados con el tema en cuestión, para revisar trabajos de diferentes autores correspondientes al tema de estudio y para llegar a conclusiones en cuanto a los valores de los indicadores económicos financieros obtenidos.

\section{Investigación de campo:}

Este término según el Manual de Trabajos de Grado, de Especialización y Maestrías y Tesis Doctorales, (UPEL, 2005)), es "el análisis sistemático de problemas de la realidad, con el propósito bien sea de describirlos, interpretarlos, entender su naturaleza y factores constituyentes, explicar sus causas y efectos o predecir su ocurrencia, haciendo uso de métodos característicos de cualquiera de los paradigmas de investigación conocidos" (pág. 14). 
En el presente análisis de casos el trabajo de campo se realiza en el Banco del Austro. Analizando una situación existente.

\section{Nivel o tipo de investigación}

\section{Investigación exploratoria:}

La investigación exploratoria la definen los autores (Hernández, Fernández, \& Baptista, 2003) de la siguiente manera "consiste en examinar un tema que no ha sido abordado anteriormente, y de ser el caso, se han realizado en diferentes contextos" (pág. 70). Este tipo de investigación aplica al presente análisis de casos ya que el tema del mismo se ha estudiado anteriormente, pero en diferentes contextos.

\section{Investigación descriptiva:}

Para saber en qué consiste este tipo de investigación es necesario plantear el concepto que dan los autores (Hernández, Fernández, \& Baptista, 2003), ellos plantean que "miden y evalúan diversos aspectos, dimensiones o componentes del fenómeno o fenómenos a investigar. Desde el punto de vista científico, describir es medir" (pág. 71).

Como exponen los autores antes mencionados acerca de la investigación descriptiva, en el presente análisis de casos se pone en práctica este tipo de investigación debido a que se medirán los indicadores económicos financieros del Banco del Austro, además se describirán las políticas gubernamentales que inciden en estos indicadores.

\section{Investigación Correlacional:}

Este tipo de investigación los autores (Hernández, Fernández , \& Baptista, 2003) la describen como "mide dos o más variables que se pretende ver sí están o no relacionadas en los mismos sujetos y después se analiza la correlación" (pág. 71). En el presente análisis de caso se utiliza este tipo de investigación porque se verá la relación existente entre las políticas gubernamentales y los indicadores económicos financieros del Banco del Austro.

\section{Métodos de investigación:}

Método inductivo

Los autores (Hernández, Fernández, \& Baptista, 2003) lo definen como "es un proceso por medio del cual, a partir del estudio de casos particulares, se obtienen conclusiones o leyes universales que explican o relacionan los fenómenos estudiados" (pág. 73). Se utiliza dicho método porque se está estudiando el caso particular del Banco del Austro. Mediante este estudio pudieran generalizarse los resultados con otras entidades.

\section{Método sintético}

Los mismos autores antes mencionados (Hernández, Fernández, \& Baptista, 2003) lo califican como "se relacionan hechos aparentemente aislados y se formula una teoría que 
unifica los diversos elementos" (pág. 73). Este método se utiliza para demostrar la relación existente entre las políticas gubernamentales y los indicadores económicos financieros. Ya que aparentemente estas dos variables son aisladas.

\section{Población y muestra}

En el presente análisis de casos la población que se tendrá en cuenta para el mismo son los directivos y trabajadores del Banco del Austro. Los cuáles serán entrevistados para obtener algunos de los datos que se necesitan para demostrar la relación existente entre las políticas gubernamentales y los indicadores económicos financieros del Banco. El total de la población es 28 personas que serán las entrevistadas.

\section{Muestra}

La muestra que se utilizará es el total de la población ya que es una población pequeña por lo que no es necesario hacer el cálculo de muestra. Total, de la muestra 28 personas. Para analizar los principales resultados se analizarán cada una de las variables a estudiar independientemente y después se analizará la relación existente entre las mismas.

\section{Políticas Gubernamentales (públicas)}

Para profundizar en este tema se plantearán las Políticas Gubernamentales que influyen en la rentabilidad económica financiera del Banco del Austro y se explicará en que consiste cada una de ellas. Las políticas públicas dictaminadas por el gobierno para las entidades financieras a nivel nacional, tienen el objetivo de lograr metas de bien común, transformaciones estructurales, resolución de problemas y asignación de recursos. En el código orgánico monetario y financiero se encuentran detalladas todas las políticas públicas referentes a las entidades financieras tanto públicas como privadas.

En el año 2012 se crearon 3 resoluciones para el control y manejo de la liquidez del sistema financiero privado. Estas resoluciones fueron

$\checkmark$ La resolución $027-2012$ el 20 de junio:

Esta resolución dispuso un mayor aporte al fondo de liquidez:

La resolución 028-2012 el 11 de julio

Esta resolución aumentó el encaje bancario de $2 \%$ a $4 \%$, incrementó el coeficiente de liquidez doméstica de $45 \%$ a $60 \%$, y aumentó las reservas mínimas de liquidez que las instituciones financieras privadas debieron constituir localmente.

$\checkmark$ La resolución 029-2012 del 11 de julio.

La Regulación 029-2012 acaparó la llegada de divisas al país (empezó a regir desde el 1 de noviembre de 2012). Las transferencias de divisas provenientes del exterior por cualquier concepto, que las personas naturales y jurídicas solicitan a las instituciones financieras internacionales a través de las nacionales, primero deben ser acreditadas en 
las cuentas que mantienen las instituciones financieras del país en el BCE. Luego, las instituciones financieras nacionales entregarán estas divisas en efectivo, acreditación en cuenta $u$ otros medios de pago, a sus clientes beneficiarios.

Como se evidencia en las tres resoluciones anteriores los dueños de los Bancos privados se afectan grandemente con las mismas.

$\checkmark$ COSEDE-DIR-2013-00.

Esta resolución plantea lo que tiene que aportar los Bancos privados anualmente a la prima fija.

$\checkmark$ No. JB-2012-2239

En esta resolución se exponen algunos cambios en el fondo de liquidez del sistema ecuatoriano.

$\checkmark$ No. JB-2012-2237

Esta política habla de quienes pueden ser o no accionistas de las instituciones del sistema financiero

$\checkmark$ No. JB-2011-1906

En esta política se plantean las normas de sanciones del sistema de seguros de depósitos, así como cuáles son las acciones consideradas infracciones de las entidades financieras y su correspondiente sanción. Esta política es una forma de controlar de que todos los Bancos realicen el aporte al fondo de liquidez nacional en fechas específicas y además controlan la cantidad de dinero que se maneja en los Bancos privados

\section{$\checkmark$ Resolución COSEDE-DIR-2014-001}

En esta resolución se plantea aumentar el valor máximo por concepto de cobertura de seguro de \$28000 dólares que era en el 2011 a \$32000 dólares a partir del 2014. Esto beneficia a los clientes en caso de que el Banco quiebre, pero perjudica al Banco porque tendría que pagar más efectivo.

Tabla 1

Resumen de las políticas gubernamentales

\begin{tabular}{lll}
\hline Política & Año & Clasificación \\
\hline Resolución 027-2012 & 2012 & Regulatoria \\
Resolución 028-2012 & 2012 & Regulatoria \\
Resolución 029-2012 & 2012 & Redistributiva \\
COSEDE-DIR-2013-00 & 2013 & Regulatoria \\
No. JB-2012-2239 & 2012 & Redistributiva \\
\hline
\end{tabular}




\section{Tabla 1}

Resumen de las políticas gubernamentales (continuación)

\begin{tabular}{lll}
\hline Política & Año & Clasificación \\
\hline No. JB-2012-2237 & 2012 & Regulatoria \\
No. JB-2011-1906 & 2011 & Regulatoria \\
COSEDE-DIR- 2014-001 & 2014 & Distributiva \\
\hline
\end{tabular}

Fuente: Código Orgánico Monetario

\section{Rentabilidad económica financiera}

Para realizar el análisis de la rentabilidad económica financiera se calcularán los indicadores económicos financieros por cada uno de los años de análisis y se realizará una comparación del comportamiento de estos en el periodo.

Para calcular los indicadores financieros en cada uno de los años, se utilizó la información contable del balance general. Esta información podrá verificarse en los anexos.

Tabla 2

\begin{tabular}{cccccc}
\hline \multicolumn{5}{c}{ Resumen de los indicadores por año } \\
\hline Indicadores & 2010 & 2011 & 2012 & 2013 & 2014 \\
Liquidez & 0.31 & 0.28 & 0.30 & 0.30 & 0.28 \\
Solvencia neta & 1.09 & 1.10 & 1.10 & 1.09 & 1.09 \\
$\begin{array}{c}\text { Endeudamiento } \\
\text { del activo }\end{array}$ & 0.92 & 0.90 & 0.91 & 0.91 & 0.91 \\
$\begin{array}{c}\text { Endeudamiento } \\
\text { patrimonial }\end{array}$ & 10.78 & 9.8 & 9.91 & 10.21 & 10.48 \\
ROE & 0.17 & 0.16 & 0.13 & 0.099 & 0.098 \\
ROA & 0.014 & 0.015 & 0.011 & 0.008 & 0.008 \\
\hline
\end{tabular}

Fuente: Banco del Austro

\section{Liquidez}

La liquidez en el Banco del austro en el año 2010 es bastante baja pues el valor de 0.31 no es un valor recomendable. Esto significa que la capacidad que tiene el Banco de pagar sus deudas a corto plazo no es buena. Lo que se interpreta que por cada dólar el Banco cuenta con 0,31 dólar.

En el año 2011 es más baja que en el 2010 pues el valor de 0.28 no es un valor recomendable. Además de que de un año a otro disminuyó. En el año 2012 es más baja que en el 2010 aunque aumentó con respecto al 2011. Pero siguen siendo valores bajos. 
En el año 2013 es la misma del año 2012 y más baja que en el 2010 con un valor de 0.30 . Corroborando así los bajos valores que viene arrastrando el Banco en los últimos años. En el año 2014 es la misma del 2011 y más baja que en el 2010 donde se puede apreciar que son los valores más bajos de los años de análisis. A pesar de que la tendencia de los valores de liquidez es a la fluctuación. En el año 2014 se registró el valor más bajo.

\section{Solvencia neta}

Este indicador muestra que la empresa en los años 2010, 2013 y 2014 cuenta con 1.09 dólares para pagar cada dólar de la deuda en el año. Pero en los años 2011 y 2012 el valor era de 1.10 dólares que son valores bajos y se han mantenido constantes en los 5 años de análisis.

\section{Endeudamiento del Activo}

Este indicador muestra que en el año 2010 el 92\% del activo total es financiado por el pasivo total, el resto se realiza por intermedio del patrimonio del Banco. Pero este porciento disminuyó en los años posteriores siendo del $90 \%$ en el 2011 y del $91 \%$ en los años 2012, 2013 y 2014. Esto da una tendencia de mantenerse constante o sea que el Banco está en una etapa de estancamiento.

\section{Endeudamiento patrimonial}

Debido a que este valor es bastante alto entonces las posibilidades del Banco de obtener fondos adicionales mediante préstamos es muy poco probable. A pesar de que el mayor valor fue el del año 2010 comenzó a disminuir en el 2011 y volvió a aumentar en el periodo del 2012 al 2014.

\section{Rentabilidad financiera (ROE) y rentabilidad económica (ROA)}

Como el ROE > ROA el efecto de apalancamiento es positivo esto significa que la tasa de rendimiento que se alcanza sobre los activos del Banco, es mayor a la tasa de interés que se paga por los fondos obtenidos en los préstamos. Esto sucede en todos los años de análisis, pero los valores de estos dos indicadores disminuyen por cada uno de los años de análisis. Acerca de los resultados obtenidos en cuanto a indicadores financieros se puede concluir que los mismos se mantienen relativamente constantes, pero con una tendencia a la disminución. Además de que no son lo suficientemente favorables.

\section{Relación entre las variables de estudio.}

Para demostrar la relación existente entre las variables de estudio se realizó una encuesta a los directivos y trabajadores del Banco del Austro y utilizando la prueba de chi cuadrado demostrar que las políticas gubernamentales influyen en la rentabilidad del Banco del Austro.

\section{Verificación de la hipótesis.}

\section{Planteamiento de la hipótesis.}


Ho: Las políticas gubernamentales no inciden sobre la rentabilidad del Banco del Austro.

H1: Las políticas gubernamentales inciden sobre la rentabilidad del Banco del Austro.

\section{Nivel de significancia}

El nivel de significancia es del 0.05 , lo que provoca que exista un $95 \%$ de que se cumpla la hipótesis nula.

\section{Especificación del estadístico}

El estadístico es:

$$
\mathrm{x}^{2}=\frac{\sum(\mathrm{O}-\mathrm{E})^{2}}{\mathrm{E}}
$$

\section{DONDE:}

$$
\begin{aligned}
& X^{2}=\text { chi cuadrado } \\
& O=\text { Frecuencia observada } \\
& E=\text { Frecuencia esperada }
\end{aligned}
$$

\section{Determinación de los grados de libertad}

Para determinar los grados de libertad es importante saber cuántos aspectos se evalúan, conociendo esto los grados de libertad son el total de aspectos menos 1. Los aspectos a tener en cuenta son:

$\checkmark$ Incidencia de las políticas Gubernamentales en la rentabilidad

$\checkmark$ Incidencia de otros factores en la rentabilidad

Como los aspectos a tratar son 2 entonces los grados de libertad tienen valor 1

CRITERIO: Acepta la hipótesis nula si: $\mathrm{x}_{\mathrm{c}}^{2} \leq \mathrm{x}_{\mathrm{t}}^{2}=3.84$

\begin{tabular}{|c|c|c|c|c|}
\hline \multicolumn{5}{|c|}{ Frecuencias Observadas } \\
\hline & & \multicolumn{3}{|c|}{ Rentabilidad } \\
\hline & & $\mathrm{SI}$ & NO & $\Sigma$ \\
\hline & Inciden las políticas & 37 & 19 & 56 \\
\hline & Inciden otros factores & 34 & 78 & 112 \\
\hline$\Sigma$ & & 71 & 97 & 168 \\
\hline
\end{tabular}

Donde $\mathrm{x}_{\mathrm{c}}^{2}$ es el valor del Chi cuadrado calculado $\mathrm{y} \mathrm{x}_{\mathrm{t}}^{2}$ es el Chi de la tabla.

Frecuencias Observadas y frecuencias esperadas

Tabla 3

Fuente: Encuesta 
Para tabular los valores en la tabla de frecuencias observadas para otros indicadores se sumaron los resultados de las preguntas 3, 4, 6, 7. Estas fueron seleccionadas pues en ellas se recogen los datos de los otros factores que inciden en la rentabilidad del Banco. Para calcular los valores de la incidencia de las políticas en la rentabilidad se tomaron los valores de las preguntas 5, 8. Para tabular los valores de la pregunta 5 es necesario aclarar que las respuestas positivas significan que las políticas no inciden y las respuestas negativas significan que las políticas si inciden.

Tabla 4

\begin{tabular}{ccc}
\hline \multicolumn{3}{c}{ Frecuencias Esperadas } \\
\hline \multicolumn{3}{c}{ Rentabilidad } \\
& SI & NO \\
Incidencia de las & 23.7 & 32.3 \\
Inciden otros factores & 47.3 & 64.7 \\
\hline
\end{tabular}

Fuente: Encuesta

De acuerdo a los valores obtenidos se rechaza la hipótesis nula $\left(\mathrm{H}_{0}\right)$, esto significa que se acepta $\mathrm{H}_{1}$ y al aceptar $\mathrm{H}_{1}$ se está afirmando que las políticas gubernamentales inciden en la rentabilidad del Banco del Austro.

\section{Conclusiones}

- La investigación demostró que las políticas económicas gubernamentales incidieron en los indicadores económicos de la institución financiera en análisis, se concluye que las resoluciones adoptadas por el gobierno afectaron la liquidez del banco, puesto que dictan que los bancos deberán aportar un mayor porcentaje a los fondos de liquidez.

- Otras de las medidas que perjudicaron al banco ha sido el incremento del encaje bancario de un $2 \%$ a un $4 \%$ reduciendo de esta forma la proporción de dinero con que el Banco puede desarrollar sus operaciones.

- Como se hizo evidente en los cálculos de los indicadores financieros del Banco del Austro para el lapso de estudio, los resultados no fueron alentadores para la organización, puesto que la mayor parte de los indicadores se mantenían constantes con tendencia decreciente como se ha demostrado en el análisis del ROE y ROA.

- Se concluye también que las políticas gubernamentales económicas en el lapso de estudio tuvieron una incidencia directa en los valores de los indicadores financieros del Banco del Austro.

\section{Referencias bibliográficas}

Aguilera, C. I. (2000). Un enfoque gerencial de la teoría de las restricciones. . Estudios Gerenciales, 53-70. 
Allardt, E. (1996). Tener, amar, ser: una alternativa al modelo sueco de investigación. Mexico: En M. Nussbaum y A. Sen.

Andrade , S. (2005). Diccionario de Economía. Tercera Edición. Quito: Editorial Andrade.

Asamblea Nacional. (2014). Codigo Organico monetario y financiero. Quito.

Baena Toro, D. (2010). Análisis Financiero, Primera Edición,. Colombia: Ecoe Ediciones.

Bodie , Z., \& Merton , R. (2003). Finanzas. Prentice Hall - Pearson Education.

Braley, R., \& Myers, S. (2002). Principios de Finanzas Corporativas, 3ra. Edic. Caracas: Mc Graw -Hill.

Cortés Romero, A., Rayo Cantón, S., \& Lara Rubio, J. (2011). Un Modelo ExplicativoPredictivo de la Rentabilidad Financiera de las Empresas en los Principales Sectores Económicos Españoles. Granada.: XVI CONGRESO AECA,.

Delgado Godoy , L. (2009). Las políticas públicas. El ciclo de las políticas públicas. Castilla- L aMancha: Consejería de Administraciones Públicas.

Derkach , D. (1986). Análisis de la Actividad Económica de las Empresas Industriales. La Habana: Pueblo y Educación.

Fernández, N. (2013). Medir y cuidar la rentabilidad. Pymes No 106, Seccion dinero.

Fontalvo Herrera , T., Mendoza Mendoza , A., \& Morelos Góme, J. (2011). Evaluación del impacto de los sistemas de gestión de la calidad en la liquidez y rentabilidad de las empresas de la Zona Industrial de Mamonal. Revista Virtual Universidad Católica del Norte. No. 34, 314 - 341.

Fontalvo Herrera, T. J. (2014). Application of discriminant analysis to assess productivity as a result from the BASC certification in Cartagena companies. Contaduría y administración,, 43 - 62.

Foulke, R. (1973). Análisis práctico de estados financieros. México: UTEHA.

GITMAN, L. (1997). Princípios de Administración Financeira. 7. ed. São Paulo: Harbra.

Hernández, R., Fernández, C., \& Baptista. (2003). Metodología de la Investigación. México: McGraw- Hill Interamericana de México.

Herrera, L., \& Medina, A. (2004). Tutoría de la Investigación científica. Quito: Dimerino.

Horngren, C. (1996). Contabilidad de costos : un enfoque gerencial. México: PrenticeHall Hispanoamericana. 
Horngren, C., Sundem, G., \& Stratton, W. (2006). Contabilidad admnistrativa. 13ra edicion. Mexico: Pearson educación.

Jami Negrete Héctor Adeodato, H., \& Karolys Tovar , M. (2012). LA FALTA DE APLICACIÓN DE POLÍTICAS DE ESTADO EN EL CENTRO DE REHABILITACIÓN SOCIAL DE LATACUNGA. Latacunga: UTC.

Jaramillo Bernita, E. (2015). ANÁLISIS Y EVALUACIÓN DEL IMPACTO DE LA RESOLUCIÓN \# 116 DEL COMITÉ DE COMERCIO EXTERIOR (COMEX) APLICADAS A LAS IMPORTACIONES EN EL ECUADOR. Quito: PUCE.

Knoepfel, P. (2007). Public Policy Analysis. Inglaterra: University of Bristol.

Morelos Gómez, J., Fontalvo Herrera , T., \& de la Hoz Granadillo , E. (2012). Análisis de los indicadores financieros en las sociedades portuarias de Colombia. Entramado, 14 - 26.

Núñez, M. d. (2004). Desarrollo socioeconómico (Primera Edición ed.). México: Editorial Ariel S.A.

Olavarría Gambi, M. (2007). Conceptos Básicos en el Análisis de Políticas Públicas. Chile: INAP.

ONU. (1990). Informe Anual de Desarrollo Humano. ONU.

Quishpe Quispe , N. (2014). LA POLÍTICA PÚBLICA DE ASISTENCIA SOCIAL A TRAVÉS DEL CRÉDITO DE DESARROLLO HUMANO Y SU INCIDENCIA EN LAS CONDICIONES DE VIDA DE LOS BENEFICIARIOS”. Ambato: UTA.

Rodriguez Montenegro, E., \& Yépez Andino, A. (2014). ANÁLISIS DE LAS POLÍTICAS PÚBLICAS DIRIGIDAS A LA INSERCIÓN SOCIO-LABORAL DE PERSONAS CON DISCAPACIDAD EN EL ECUADOR. Quito: UCE.

Ruiz Lopez, D., \& Cadenas Ayala, C. E. (2013). ¿Que es una politica publica? Revista juridica, 1-33.

Ruiz Porras , A., \& Steinwascher, W. (2007). Gobierno corporativo, diversificación estratégica y desempeño empresarial en México. Monterrey, México: FLACSO.

Sánchez Pérez, I. (2004). México: Editorial Ariel S.A.

Schaffer, R. (2000). Desarrollo Social (Segunda Edición ed.). México: Editores S.A.

Superintendencia de Bancos y Seguros. (2011). Resolución No. JB-2011-1906. Quito: Republica del Ecuador .

Superintendencia de Bancos y Seguros. (2012). Resolucion 027- 2012. Quito: Republica del Ecuador. 
superintendencia de Bancos y Seguros. (2012). Resolucion 029-2012. Quito: Republica del Ecuador.

Superintendencia de Bancos y Seguros. (2012). Resolucion No. JB-2012-2239. Quito: Republica del Ecuador.

Superintendencia de Bancos y Seguros. (2012). Resolucion No.JB-2012-2237. Quito: Republica del Ecuador .

Superintendencia de Bancos y Seguros. (2013). COSEDE-DIR-2013-00. Quito: Republica del Ecuador.

Superintendencia de Bancos y Seguros. (2014). COSEDE-DIR-2014-001. Quito: Republica del Ecuador.

Tamayo M. (2004). Análisis de las políticas públicas. En La Nueva Administración Pública. Madrid: Universidad Complutense de Madrid.

UPEL. (2005). Manual de trabajos de grado de especialización y maestría y tesis doctorales. Caracas: Universidad Pedagógica Experimental Libertador.

Vásquez, X., Guerra , A., \& Ahmed., I. (2008). Aplicación de métodos multivariados: una respuesta a las limitaciones de los ratios financieros. Revista de la Universidad de Granma. Vol. $24 \mathrm{~N}^{\circ}$ 114, 44.

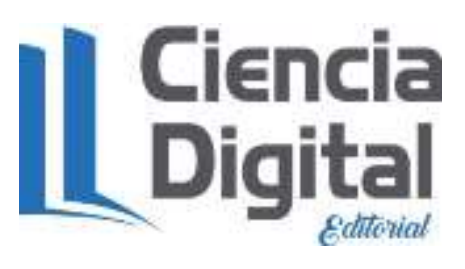




\section{PARA CITAR EL ARTÍCULO INDEXADO.}

Guerrero Freire, E. I., Santana Torres, A. A., Ibáñez Jacome, S. S., \& Flores Abarca, L. I. (2021). Evaluación financiera del Banco del Austro y análisis del impacto de las regulaciones bancarias decretadas por el Gobierno Ecuatoriano en el periodo 2010-2014. AlfaPublicaciones, 3(3.1), 152-174. https://doi.org/10.33262/ap.v3i3.1.84

\section{Ligital}

El artículo que se publica es de exclusiva responsabilidad de los autores y no necesariamente reflejan el pensamiento de la Revista Alfa Publicaciones.

El artículo queda en propiedad de la revista y, por tanto, su publicación parcial y/o total en otro medio tiene que ser autorizado por el director de la Revista Alfa Publicaciones.
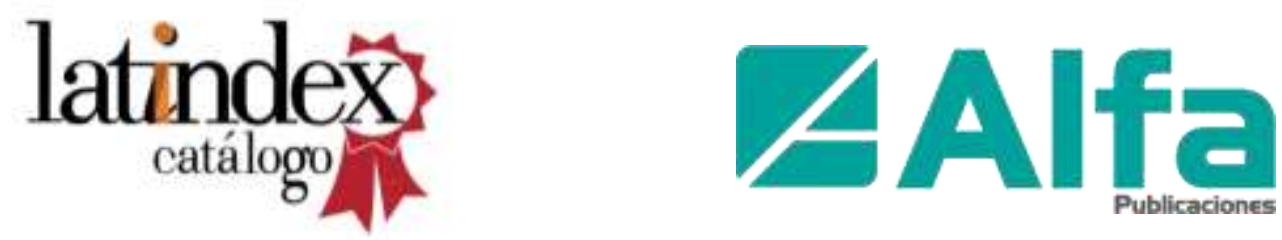